\title{
Fundamental solutions and singular shocks in scalar conservation laws
}

\author{
Emmanuel CHASSEIGNE \\ Laboratoire de Mathématiques \\ Université de Tours \\ France \\ echasseigne@univ-tours.fr
}

Recibido: 8 de Octubre de 2002

Aceptado: 5 de Febrero de 2003

\begin{abstract}
We study the existence and non-existence of fundamental solutions for the scalar conservation laws$$
u_{t}+f(u)_{x}=0
$$

related to convexity assumptions on $f$. We also study the limits of those solutions as the initial mass goes to infinity. We especially prove the existence of so-called Friendly Giants and Infinite Shock Solutions according to the convexity of $f$, which generalize the explicit power case $f(u)=u^{m}$. We introduce an extended notion of solution and entropy criterion to allow infinite shocks in the theory, and the initial data also has to be understood in a generalized sense, since locally infinite measures appear.
\end{abstract}

2000 Mathematics Subject Classification: 35L60, 35L67.

Key words: Conservation laws, fundamental solutions, singular solutions

\section{Introduction}

In this paper, we study the Fundamental Solutions of the scalar conservation law

$$
u_{t}+f(u)_{x}=0, \quad(x, t) \in Q=\mathbb{R} \times \mathbb{R}_{+},
$$

that is, we consider nonnegative solutions taking the initial data $u_{0}(x)=c \delta_{0}(x)$, $c>0$. Of interest here is the existence of such solutions according to the properties of $f$, and the limit as $c$ tends to $+\infty$ of such solutions.

The study of fundamental solutions of (1.1) in the case of functions $f$ which are Lipschitz up to the origin has been done by Tai-Ping Liu and Michel Pierre in [11]. While uniqueness of solutions holds without any other restriction on $f$, some convexity 
assumption on $f$ is needed in order to get existence of solutions with measure initial data. Moreover, the authors study the long-time behaviour of arbitrary solutions, which appears to be closely related to fundamental solutions, an important idea that has also been applied to a number of other situations.

Concentrating on fundamental solutions, we want to extend the study in [11] to other functions $f$ which can be concave or neither convex nor concave. Moreover, we are interested in seeking the limit as $c \rightarrow \infty$ of the fundamental solutions with mass $c>0$. This limit, as well as the existence of fundamental solutions are linked to the properties of $f$, and as we shall see, some special solutions presenting "infinite discontinuities" may appear for concave f's.

Before explaining our approach and our results, let us mention that such investigations have been done by a number of authors in parabolic equations. We refer for instance to the works of Kamin, Brezis, Peletier, Terman, Vázquez... (see [15] and the references therein), and especially the paper of Vázquez and Véron [14], to which the present work is related. In the case of parabolic equations, those solutions are not necessarily classical (existence of free boundaries in some situations), but at least they are continuous. It is also important to recall that fundamental solutions and very singular solutions give the asymptotic behaviour of general weak solutions. Let us also mention that fundamental solutions for conservation laws with absorption (typical example of balance laws) were considered by F. Guarguaglini [8] and Y. Hongjun [9], but we will restrict ourselves to the purely conservative equation here.

Going back to hyperbolic equations like (1.1), it is well-known that solutions are not necessarily continuous, leading to the concept of "shock". A shock is a discontinuity of the solution, and the presence of such shocks which propagate along a curve $\Gamma$ implies to find a suitable notion of solution. Let us only mention here two basic facts, referring to the introduction for precise statements :

i) The notion of solution has to be understood in a weak sense, which implies that some conditions on the discontinuities have to be fulfilled in order to get an acceptable (weak) solution. These are the well-known Rankine-Hugoniot conditions on $\Gamma$, which can be expressed in different forms.

ii) Even with this good notion of solution, one needs an additional assumption in order to get uniqueness of weak solutions. On the point of view of physical interpretations, the "good" solution can be obtained by the well-known vanishing viscosity method, which consists in solving the non-degenerate parabolic equation

$$
u_{t}+f(u)_{x}=\varepsilon u_{x x}, \quad \varepsilon>0
$$

and passing to the limit as $\varepsilon \rightarrow 0$, in order to get a solution of (1.1).

A more general way to get uniqueness relies on the notion of entropy solution (see preliminaries for a precise definition). This is the notion of solution we will take here, but we shall give also example of weak solutions which are not entropic,i.e., solutions with non entropic shocks. For simplicity, let us mention that in the case of convex 
functions $f$, a "good shock" satisfies

$$
[u]=u_{-}-u_{+}<0 \quad \text { on } \quad \Gamma,
$$

with the usual notation $u_{ \pm}$for the left- and right-side spatial limits of $u$ at the shock. The contrary holds for concave functions $f$.

Motivations and aims of the paper. As we said, it was proved by Liu and Pierre [11] that if $f$ is convex, there exists a unique Entropic Fundamental Solution of (1.1) for any $c>0$. At least in the case $f(u)=u^{m}, m>1$, the explicit form is known:

$$
u_{c}=\left\{\begin{array}{cl}
(x / m t)^{1 /(m-1)}, & 0 \leqslant x \leqslant \xi(t), \\
0, & x>\xi(t),
\end{array}\right.
$$

where $\xi(t)=m(c /(m-1))^{(m-1) / m} t^{1 / m}$. Then it is clear that the limit as $c \rightarrow \infty$ of $u_{c}$ is the following singular solution:

$$
U_{1}(x, t)=\left(\frac{x}{m t}\right)^{1 /(1-m)} .
$$

This solution is a kind of Friendly Giant, as named by Dahlberg and Kenig [6] in the context of porous medium equations: considered in $Q_{+}=\left(\mathbb{R}_{+}\right)^{2}$, it is a classical solution with infinite initial data, but it satisfies the zero Dirichlet condition on the boundary $\{x=0, t>0\}$.

More interesting for our purpose is the concave case, where a model example is $f(u)=u^{m}, 0<m<1$. The explicit self-similar solution is not a Friendly Giant:

$$
U_{2}(x, t)=\left(\frac{m t}{x}\right)^{1 /(1-m)} \quad \text { in } Q_{+},
$$

and $U_{2} \equiv 0$ in $Q_{-}=\mathbb{R}_{-} \times \mathbb{R}_{+}$. This other special solution is alike the so-called Razor Blade, already studied in the parabolic case (see [14, 2, 3]) and existence of this special solution is characteristic of sub-linear non-linearities. This solution has a constant singularity at $x=0$ for any time $t>0$, but begins with zero initial data for $x \neq 0$. Let us notice that the above solutions cannot be symmetrized for $x<0$ unless we relax the nonnegativity assumption (and we assume that $f$ is odd). This is a consequence of the non-invariance of the equation under the change of variable $x \rightarrow-x$. On the contrary, in the parabolic case, the corresponding solutions are even of course.

Let us also mention at this point that the initial data of $U_{2}$ itself has to be understood in a wider sense. Indeed, roughly speaking, such a solution $u$ takes on the initial data

$$
u(0)=\infty \cdot \delta_{0} \quad \text { in } \quad \mathbb{R} .
$$

This kind of initial data enters in the class of general Borel measures, i.e., nonnegative measures which are not necessarily locally finite. These measures are known to play a 
fundamental role in various equations of parabolic and elliptic type since the works of various authors concerning Very Singular Solutions. Such solutions were studied by F. Guarguaglini [8] in the case of conservation laws with strong absorption. Marcus and Véron [12] provided a general setting to study solutions with traces that are Borel measures and their approach was used by the author in various other contexts (see $[1,2]$ and $[3,4]$ with J.L. Vázquez).

Our aim is to generalize these explicit examples. The first question that arises concerns the existence of fundamental solutions for general $f$ 's. Second, if such solutions exist, what is their limit as $c \rightarrow \infty$. Is it a kind of Friendly Giant, as above, or are there other possibilities of limits ? Let us also notice that in the case $f(u)=u^{m}, m>1$ (and the same for $0<m<1$ ), the fundamental solution is obtained by a suitable truncation of the Friendly Giant, i.e., a suitable shock curve. So the third question is: are the fundamental solutions obtained by truncation of some other solution in general ? Finally, what is the sense of the special solution $U_{2}$ obtained in (1.4) ? This solution has an "infinite shock" on the curve $\Gamma=\{x=0\}$ and we would like to better understand the meaning of such singular shocks.

We will thus give some precise answers to these questions, using the important fact that for any $f$, there is an explicit self-similar solution of the form $U(x, t)=\mathrm{G}(x / t)$. Self-similar solutions are well-known to exist, as a consequence of the invariancy of the equation under the change of variables $(x, t) \rightarrow(k x, k t), k \in \mathbb{R}$. In the parabolic case, the change of variable is a little bit different, but still self-similar solutions do exist. A big difference here is that we can find G explicitly since it has to satisfy the equation:

$$
\eta \mathrm{G}^{\prime}(\eta)-(f \circ \mathrm{G})^{\prime}(\eta)=0
$$

which leads formally to

$$
U(x, t)=\mathrm{G}(x / t)=\left(f^{\prime}\right)^{(-1)}(x / t)
$$

Of course, it is not clear that $\left(f^{\prime}\right)^{(-1)}$ is well-defined but we will restrict ourselves to fluxes such that $\mathrm{G}$ be well-defined at least in a real interval.

Before stating our main results, let us mention that the concave and convex cases can be related through the exchange of variables $x \leftrightarrow t$, the convex flux $f$ being transformed formally into the concave flux $f^{(-1)}$. Probably, most of the results obtained in the convex case could be directly translated to the concave case thanks to this transform, but we prefer here to present each case in a direct way to better understand the situation (see however the important remark 5.2-iii) ). Also, we shall only deal with nonnegative solutions, so that the flux function $f$ is only defined on $\mathbb{R}_{+}$. A similar study could be done with odd functions $f$ defined on the whole line, but we do not want to consider changing-sign solutions here.

Let us now briefly describe our Main results :

Extended Entropy Solutions. Under the assumptions we make in this paper, $\left(f^{\prime}\right)^{(-1)}$ is well-defined on an open interval $\mathcal{I} \subset \mathbb{R}_{+}$, and we construct the following 
function:

$$
\mathcal{U}(x, t):=\left\{\begin{array}{cl}
\left(f^{\prime}\right)^{(-1)}(x / t) & \text { in }\{x / t \in \mathcal{I}\}=Q_{\mathcal{I}}, \\
0 & \text { in } Q \backslash Q_{\mathcal{I}} .
\end{array}\right.
$$

As we shall see, this function has an infinite shock along a curve $\Gamma=\{x=\ell \cdot t\}$, for some $\ell \geqslant 0$. Thus we need to extend the notion of entropy solution to include these infinite discontinuities of the solutions. The Rankine-Hugoniot condition is then expressed in the following form on $\Gamma$ :

$$
\frac{[f(\mathcal{U})]}{[\mathcal{U}]}=\lim _{u \rightarrow \infty} \frac{f(u)}{u}=\ell=\Gamma^{\prime}(t) .
$$

The extended entropy solution $\mathcal{U}$ is a kind of Friendly Giant if $f$ is convex, and a kind of Razor Blade if $f$ is concave, which generalizes the cases $f(u)=u^{m}$.

Existence of Fundamental Solutions. The existence and non existence of fundamental solutions is closely related to the total mass carried by $\left(f^{\prime}\right)^{(-1)}$ (see exact formulation in Preliminaries Section): if this mass is infinite, we will be able to construct fundamental solutions by a suitable truncation of $\mathcal{U}$, whether $f$ is convex or concave. Moreover, as the mass $c>0$ goes to infinity, the fundamental solutions $u_{c}$ converge to $\mathcal{U}$ itself, as it is the case for powers.

Non Existence of Fundamental Solutions. If the mass carried by $\left(f^{\prime}\right)^{(-1)}$ is finite, say its value is $\mathrm{M}>0$, then the special solution $\mathcal{U}$ takes on the zero initial data in the sense of measures. Indeed, the total mass of $\mathcal{U}$ at time $t>0$ is exactly $\mathrm{M} \cdot t$. This surprising case shows that we cannot construct any fundamental solution, and that there exists a non-trivial solution starting with the zero initial data. This example seems in contradiction with the name "conservation law" attributed to (1.1). However, it is shown that $\mathcal{U}$ is in fact a solution of the equation with a forcing term:

$$
\mathcal{U}_{t}+f(\mathcal{U})_{x}=\mathrm{M} \cdot \mu_{\Gamma}(x, t),
$$

where $\mu_{\Gamma}$ is a Dirac mass which travels along the shock curve $\Gamma$. This explains the non-conservation of mass.

Viscous Approximation. It is clear that solutions having infinite shocks cannot be obtained as limits of the usual vanishing viscosity method, which consists in solving first $u_{t}+\varepsilon u_{x x}+f(u)_{x}=0$, and send $\varepsilon \rightarrow 0$. However, when $f$ is concave, it is probable that these solutions can be approached by the solutions of the following nonlinear viscous equation:

$$
u_{t}+\varepsilon f(u)_{x x}+f(u)_{x}=0 .
$$

Indeed, such an equation allows infinite values of the solutions. We prove this result in the case $f(u)=u^{m}, 0<m<1$ where explicit calculations can be done. 


\section{Preliminaries - Extended entropy solutions}

A. Notations. Throughout this paper, $f$ is a nonnegative function defined on $\mathbb{R}_{+}$, as we shall only deal with nonnegative solutions. Unless otherwise specified, we shall assume that the fluxes $f$ belong to the class

$$
\mathcal{F}=\left\{f \in \mathrm{C}^{0}([0, \infty)) \cap \mathrm{C}^{1}(0, \infty), f(0)=0, f^{\prime}>0 \text { not constant }\right\} .
$$

In fact, we could require less regularity on $f$ like locally Lipschitz, but we are not concerned with optimal regularity here. For any flux function $f \in \mathcal{F}$, the following numbers are well-defined in $\mathbb{R}_{+} \cup\{+\infty\}$ :

$$
\ell_{\text {inf }}=\inf f^{\prime}, \quad \ell_{\text {sup }}=\sup f^{\prime},
$$

and we denote by $\mathcal{I}$ the interval $\left(\ell_{\text {inf }} ; \ell_{\text {sup }}\right)$, which is not void since $f^{\prime}$ is not constant. If $f$ is either convex or concave, the function $\left(f^{\prime}\right)^{(-1)}$ is well-defined exactly on $\mathcal{I}$, so that we may consider the following integral:

$$
\mathrm{M}(f)=\int_{\mathcal{I}}\left(f^{\prime}\right)^{(-1)}(\eta) d \eta \in(0, \infty] .
$$

The numbers $\ell_{\text {inf }}, \ell_{\text {sup }}$ and the mass $\mathrm{M}(f)$ will play an important role in the following study. Now, if $f$ is either convex or concave, the following function is well-defined:

$$
\bar{U}_{\mathcal{I}}(x, t)=\left(f^{\prime}\right)^{(-1)}(x / t), \quad(x, t) \in Q_{\mathcal{I}}:=\{x / t \in \mathcal{I}\} .
$$

Clearly, $\bar{U}_{\mathcal{I}}$ is the maximal self-similar solution, in the sense that it has the biggest domain of definition among non-trivial self-similar solutions.

Remark 2.1. In general the domain $Q_{\mathcal{I}}$ is only a proper subset of $Q_{+}=\left(\mathbb{R}_{+}\right)^{2}$, a difficulty we will have to treat in the sequel. This situation happens when $f$ as a linear behaviour near 0 or near $+\infty$. In many situations, $Q_{\mathcal{I}}=Q_{+}\left(\right.$like $\left.f(u)=u^{m}, m \neq 1\right)$, but we want to work in full generality here.

To obtain a solution defined in the whole set $Q$, we construct a special solution $\mathcal{U}$ as follows:

$$
\mathcal{U}(x, t):= \begin{cases}\bar{U}_{\mathcal{I}}(x, t) & \text { in } Q_{\mathcal{I}} \\ 0 & \text { in } Q \backslash Q_{\mathcal{I}} .\end{cases}
$$

Such a solution which has infinite discontinuities at one boundary of $Q_{\mathcal{I}}$, so we will need to introduce a new concept of solution to work with $\mathcal{U}$ (see the paragraph Extended Entropy Solutions below).

We end this paragraph by mentioning that if $f$ is convex,

$$
\lim _{\eta \rightarrow \ell_{\mathrm{inf}}}\left(f^{\prime}\right)^{(-1)}(\eta)=0, \quad \lim _{\eta \rightarrow \ell_{\text {sup }}}\left(f^{\prime}\right)^{(-1)}(\eta)=+\infty,
$$


so that $\left(f^{\prime}\right)^{(-1)}$ is always integrable near $\ell_{\text {inf }}$. The contrary holds for concave functions $f$. Also, if $f$ is convex and $\ell_{\text {sup }}=+\infty$, then necessarily $\mathrm{M}(f)=+\infty$. We will come back later on this point.

B. Weak Entropy Solutions. For any $f \in \mathcal{F}$, we consider equation (1.1), and we use the well-known concept of entropy solution, which reads as follows in our setting: a nonnegative function $u \in L^{\infty}\left(0, T ; L^{1}(\mathbb{R})\right) \cap L^{\infty}((\tau, T) \times \mathbb{R})$ for any $\tau>0$ is an entropy solution of (1.1) if for any $k \in \mathbb{R}$, and any nonnegative $\phi \in \mathcal{C}_{0}^{\infty}(Q)$,

$$
\int_{0}^{T} \int_{\mathbb{R}}|u-k| \phi_{t}+\operatorname{sign}(u-k)(f(u)-f(k)) \phi_{x} \geqslant 0 .
$$

We shall not enter into details concerning the notion of entropy solution and its various formulations here, referring to $[5,7,10]$ for precise statements, but let us give a simpler formulation: throughout this paper, we only deal with regular shock curves $\Gamma \in \mathcal{C}^{1}$. Thus, in the case of convex functions $f$, the entropy solution is selected by requiring that across the shock curve $\Gamma$, the solutions goes up when moving from left to right:

$$
[u]=u_{-}-u_{+}<0 .
$$

More precisely, this means that if $\left(x_{0}, t_{0}\right) \in \operatorname{Graph}(\Gamma)$, i.e., $x_{0}=\Gamma\left(t_{0}\right)$, then

$$
\lim _{x \rightarrow\left(x_{0}\right)^{-}} u\left(x, t_{0}\right)-\lim _{x \rightarrow\left(x_{0}\right)^{+}} u\left(x, t_{0}\right)<0 .
$$

For concave functions, the contrary holds (the solution goes down when moving from left to right).

Let us now recall the existence and uniqueness result of T.P. Liu and M. Pierre [11], that we summarize in the following form:

Theorem 2.2. (T.P. Liu and M. Pierre) Let us assume that $f \in \mathcal{F}$ is locally Lipschitz up to the origin.

i) For any finite nonnegative measure $\mu$ in $\mathbb{R}$, there exists at most one nonnegative entropy solution to (1.1) such that

$$
\lim _{t \rightarrow 0} u(t)=\mu \quad \text { narrowly in } \mathbb{R} .
$$

ii) If we assume moreover that $f$ is convex then for any finite nonnegative measure $\mu$ in $\mathbb{R}$, there exists an entropy solution to(1.1)

In fact, the uniqueness result in [11] does not need other regularity assumption on $f$ that mere continuity on $[0, \infty)$ since only distribution techniques are involved in the proof. Thus we state the following extension which will be sufficient for our purpose:

Proposition 2.3. Let $f \in \mathcal{F}$ and $u, v \in \mathrm{L}^{\infty}\left(0, T ; L^{1}(\mathbb{R})\right) \cap \mathrm{L}^{\infty}((\tau, T) \times \mathbb{R})$ for any $\tau>0$ be two nonnegative entropy weak solutions of (1.1) such that

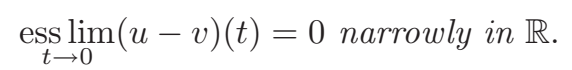


Then $u \equiv v$ in $Q=\mathbb{R} \times(0, T)$.

C. Extended Entropy Solutions. Let $f \in \mathcal{F}$ and $\Gamma: t \mapsto x=\Gamma(t)$ be a $\mathcal{C}^{1}$ curve in $Q$ that will be called the infinite shock curve. We will consider functions $u$ that can have infinite jumps from zero to $+\infty$ across $\Gamma$, like the model example constructed in (2.4).

Definition 2.1. We say that $u$ is an extended entropy solution of (1.1) with infinite shock curve $\Gamma$ if

i) The function $u$ is a continuous solution in $Q \backslash \Gamma$,

ii) The jump $[u]=u_{+}-u_{-}= \pm \infty$ satisfies the Rankine-Hugoniot condition on $\Gamma$ in the following sense:

$$
\frac{[f(u)]}{[u]}=\lim _{u \rightarrow \infty} \frac{f(u)}{u}=\frac{d \Gamma}{d t} .
$$

iii) The usual cord condition holds on $\Gamma$. In the case of convex $f$ 's, we recover that the jump has to go up from 0 to $+\infty$ when crossing $\Gamma$ from left to right (the converse for concave functions $f$ ).

Let us give an equivalent definition of the numbers $\ell_{\text {inf }}$ and $\ell_{\text {sup }}$ which will be useful to extend the Rankine-Hugoniot condition (The proof follows from standard convexity arguments, so that we omit it):

Lemma 2.4. If $f$ is convex, $\ell_{\mathrm{inf}}=\lim _{u \rightarrow 0^{+}} \frac{f(u)}{u}, \ell_{\mathrm{sup}}=\lim _{u \rightarrow+\infty} \frac{f(u)}{u}$. The reverse holds if $f$ is concave.

Then we have the following important result:

Theorem 2.5. Let $f \in \mathcal{F}$ be convex. Then the special solution $\mathcal{U}$ given by (2.4) is an extended entropy solution in $Q$, with infinite shock curve $\Gamma_{+}=\left\{x=\ell_{\text {sup }} \cdot t\right\}$.

Proof. In the set $Q_{\mathcal{I}} \subset\left(\mathbb{R}_{+}\right)^{2}$, the function $\mathcal{U}$ is obviously a solution, and it is zero outside $Q_{\mathcal{I}}$, so that we need only to check what happens on the interfaces

$$
\Gamma_{ \pm}=\left\{x=\ell_{ \pm} \cdot t\right\} .
$$

On $\Gamma_{-}$, the solution $\bar{U}_{\mathcal{I}}$ vanishes. Indeed, at a point $x / t=\ell_{\text {inf }},\left(f^{\prime}\right)^{(-1)}$ vanishes, which corresponds to the fact that $f(u) / u \rightarrow \ell_{\text {inf }}$ as $u \rightarrow 0$ (see previous Lemma). Thus there is no discontinuity on $\Gamma_{-}$.

On $\Gamma_{+}$, the solution $\bar{U}_{\mathcal{I}}$ goes to $+\infty$ as $x \rightarrow \ell_{\text {sup }} \cdot t$. Indeed, the same argument as above shows that $\left(f^{\prime}\right)^{(-1)}\left(\ell_{\text {sup }}\right)=+\infty$. Thus we have an infinite jump from $+\infty$ to 0 when crossing $\Gamma_{+}$from left to right. It remains to see that the extended version of Rankine-Hugoniot condition is satisfied. This comes from the fact that on $\Gamma_{+}$,

$$
\left(\Gamma_{+}\right)^{\prime}=\ell_{\mathrm{sup}}=\lim _{u \rightarrow+\infty} \frac{f(u)}{u},
$$


so that $\mathcal{U}$ is indeed an extended entropy solution in the whole set $\mathbb{R}^{2}$.

In a similar way one can prove the following Theorem in the concave case. Since the proof is the same, we omit it.

Theorem 2.6. Let $f \in \mathcal{F}$ be concave. Then the special solution $\mathcal{U}$ given by (2.4) is an extended entropy solution in $Q$, with infinite shock curve $\Gamma_{-}=\left\{x=\ell_{\inf } \cdot t\right\}$.

Remark 2.7. In the case $f$ convex, this solution can be viewed as a Friendly Giant in the subset $Q_{\mathcal{I}}$. However, unless $\ell_{\text {sup }}=+\infty$, the initial data of $\mathcal{U}$ is not $+\infty$ everywhere in $\mathbb{R}_{+}$. Thus, it is not a Friendly Giant in all $Q_{+}$. In the concave case, we will explain later on what is the initial data taken by $\mathcal{U}$, which can be either " $+\infty \delta_{0}$ ", or 0 in the sense of measures.

\section{The convex case}

Throughout this section, we will assume that $f$ is convex, and we make the further assumption that

$$
\mathrm{M}(f)=\int_{\mathcal{I}}\left(f^{\prime}\right)^{(-1)}(\eta) d \eta=+\infty .
$$

We then prove that the fundamental solutions are given explicitly, and that their limit (as the mass goes to infinity) is the special solution $\mathcal{U}$. As was said, it should be noticed that if $f$ is convex and $\ell_{\text {sup }}=+\infty$, then $\mathrm{M}(f)=+\infty$.

We begin with the following result:

Theorem 3.1. Let $f \in \mathcal{F}$, convex and such that $\mathrm{M}(f)=\infty$. Then the Entropic Fundamental Solution $u_{c}$ with mass $c>0$ is given by

$$
u_{c}(x, t)=\left\{\begin{array}{cl}
\mathcal{U}(x, t) & \text { if } \ell_{\mathrm{inf}} \cdot t \leqslant x \leqslant \xi_{c}(t) \\
0 & \text { if } x<\ell_{\mathrm{inf}} \cdot t \text { or } x>\xi_{c}(t)
\end{array}\right.
$$

where $\mathcal{U}$ is defined by $(2.4)$ and $\xi_{c}(t)$ is defined implicitly by the condition

$$
\forall t>0, \quad \int_{\ell_{\mathrm{inf}} \cdot t}^{\xi_{c}(t)} \mathcal{U}(x, t) d x=c .
$$

Proof. Let us consider the function

$$
(y, t) \mapsto \mathrm{K}(y, t)=\int_{\ell_{\mathrm{inf}} \cdot t}^{y} \mathcal{U}(x, t) d x,
$$

and let us notice that $\mathcal{U}(t)$ is always integrable near $x=\ell_{\text {inf }} \cdot t$ since it is continuous and vanishes at this point. Then $\mathrm{K}$ is well-defined in $Q_{+}=\left(\mathbb{R}_{+}\right)^{2}$, and we have $\partial_{y} \mathrm{~K}(y, t)=\mathcal{U}(y, t)=\left(f^{\prime}\right)^{(-1)}(y / t)$, which is never zero for $t>0$. 
Since $\mathrm{M}(f)=\infty$, we know that $\mathrm{K}(y, t) \rightarrow+\infty$ as $y \rightarrow \ell_{\text {sup }} t$. Thus, for any $c>0$, there exists a function $t \mapsto \xi_{c}(t)$ defined implicitly by

$$
\mathrm{K}\left(\xi_{c}(t), t\right)=c .
$$

Moreover, the function $\xi_{c}$ is obviously continuous and non-decreasing. Indeed, the convexity of $f$ implies that $\left(f^{\prime}\right)^{(-1)}$ is non-decreasing, and thus $\mathcal{U}$ is decreasing in the $t$-variable. Also, $\xi_{c}$ is differentiable everywhere in $(0, \infty)$, with

$$
\xi_{c}^{\prime}(t)=\frac{\partial_{t} \mathrm{~K}}{\partial_{y} \mathrm{~K}}\left(\xi_{c}(t), t\right)
$$

Now, $\partial_{y} \mathrm{~K}(y, t)=\mathcal{U}(y, t)$ and let us compute

$$
\partial_{t} \mathrm{~K}(y, t)=\int_{0}^{y} \partial_{t} \mathcal{U}(x, t) d x=\int_{0}^{y} \partial_{x} f(\mathcal{U})(x, t) d x=f(\mathcal{U})(y, t),
$$

since $f(\mathcal{U})(0, t)=f(0)=0$. Thus at the point $\left(\xi_{c}(t), t\right) \in \operatorname{Graph}\left(\xi_{c}\right)$ we obtain

$$
\xi_{c}^{\prime}(t)=\frac{f(\mathcal{U})\left(\xi_{c}(t), t\right)}{\mathcal{U}\left(\xi_{c}(t), t\right)}
$$

which expresses exactly the Rankine-Hugoniot condition on the shock curve $\operatorname{Graph}\left(\xi_{c}\right)$, since $u_{c}$ is zero at the right of the shock.

Remark. Since $\mathcal{U}$ is zero in the set $\left\{x \geqslant \ell_{\text {sup }} \cdot t\right\}$, the shock curve $\xi_{c}(t)$ lies necessarily above the infinite shock curve $\Gamma$. In the model example $f(u)=u^{m}, 0<m<1$, the shock curve $\xi_{c}(t)$ looks like $t^{1 / m}$, which indeed lies above $\Gamma=\{t=0\}$ (in this case). In fact it is clear that $\operatorname{Graph}\left(\xi_{c}\right)$ remains between the lines $\left\{x=\ell_{\text {inf }} \cdot t\right\}$ and $\left\{x=\ell_{\text {sup }} \cdot t\right\}$ for any $c>0$. Also, if $\ell_{\text {sup }}<+\infty$, the initial value of $\mathcal{U}$ is zero for $x \neq 0$. In fact, the exact initial trace of $\mathcal{U}$ may be described as " $+\infty \delta_{0}$ " in this case (see next Section).

Corollary 3.2. Let $f \in \mathcal{F}$, convex and such that $\mathrm{M}(f)=+\infty$. Then the limit of the entropic fundamental solutions as the mass c goes to $\infty$ is exactly the special solution:

$$
\lim _{c \rightarrow \infty} u_{c}(x, t)=\mathcal{U}(x, t)
$$

Moreover, the shock curves $\left\{x=\xi_{c}(t)\right\}$ converge to the infinite shock curve $\Gamma=\{x=$ $\left.\ell_{\text {sup }} \cdot t\right\}$.

Proof. From the explicit formula for $u_{c}$, it is enough to prove the convergence of the shock curves to $\Gamma$. In fact, by (3.2), we see that as $c \rightarrow \infty, \xi_{c}(t)$ has to go pointwise to $\Gamma(t)=\ell_{\text {sup }} t$, otherwise the left-side integral would remain bounded. This proves that the shock curves converge to $\Gamma$. 
Remark 3.3. We point out that we have proved the existence of fundamental solutions provided $f$ is convex and $\mathrm{M}(f)=+\infty$. In some sense, this result is an improvement of [11, Prop. 2.1], at least for Dirac initial data. Indeed, under the following assumption:

$$
\exists \alpha \in(0,1) \text { such that } f^{1-\alpha} \text { is convex, }
$$

Liu and Pierre prove that for any bounded measure $\mu$, there exists a unique solution with initial trace $\mu$. But clearly, our assumptions are a little bit more general, since (3.3) implies that $f$ is convex and strictly super-linear at infinity, so that $\mathrm{M}(f)=+\infty$. In fact, the convexity assumption on $f$ is not crucial in our method. It is only a simplification in order to have $\left(f^{\prime}\right)^{(-1)}$ well-defined, so we are able to construct fundamental solutions under more general assumptions on $f$, provided $\mathrm{M}(f)=+\infty$ (see also Comments Section).

\section{The Concave Case}

We consider now the case $f$ concave. Here also, the properties of self-similar solutions and existence of fundamental solutions are closely related to the convergence of the following integral:

$$
\mathrm{M}(f)=\int_{\mathcal{I}}\left(f^{\prime}\right)^{(-1)}(\eta) d \eta \in(0, \infty]
$$

In this section, we will restrict our study to functions $f \in \mathcal{F}$ that are concave and such that $\mathrm{M}(f)=+\infty$. Under this integral condition, we can use a similar argument as we did in the convex case. But the special solution constructed in (2.4) has a totally different behaviour near $t=0$, that has to be understood in a wider sense than usual. We describe below this kind of initial data.

Borel Measures. We use this notion, as recently developed by Marcus and Véron $[12,13]$ : any Borel measure $\nu$ may be written as $\nu=(\mathcal{S}, \mu)$, where $\mathcal{S}$ is a closed subset of $\mathbb{R}^{N}$ and $\mu \geqslant 0$ is a Radon measure on $\mathcal{R}=\mathbb{R}^{N} \backslash \mathcal{S}$. The set $\mathcal{S}$ is defined as follows:

$$
\mathcal{S}=\left\{x \in \mathbb{R}^{N} \mid \forall r>0, \quad \nu\left(B_{r}(x)\right)=+\infty\right\} .
$$

Thus, a Radon measure is a Borel measure with $\mathcal{S}=\emptyset$ and the simplest example of Borel measure which is not locally finite is given by the measure $+\infty \cdot \delta_{0}$, which can be written $(\{0\}, 0)$. This last measure is natural for our considerations since it is the monotone limit of the $c \delta_{0}$, as $c \rightarrow \infty$.

Borel Trace. Now, we say that a solution $u$ takes on the initial trace $\nu=(\mathcal{S}, \mu)$ if $u(t)$ converges weakly in measure to $\mu$ on $\mathcal{R}=\mathbb{R}^{N} \backslash \mathcal{S}$, i.e., for any $\varphi \in \mathrm{C}_{0}(\mathcal{R})$,

$$
\int_{\mathbb{R}^{N}} u(x, t) \varphi(x) d x \rightarrow \int_{\mathbb{R}^{N}} \varphi(x) d \mu(x) \text { as } t \rightarrow 0
$$


and for any $y \in \mathcal{S}$, any $r>0$,

$$
\int_{B_{r}(y)} u(x, t) d x \rightarrow+\infty \text { as } t \rightarrow 0 .
$$

In the present situation however, we shall restrict ourselves to initial data of the form $c \delta_{0}$, for some $c \in[0, \infty]$.

Our first result shows that the special solution $\mathcal{U}$ with infinite shocks on the axis $\left\{x=\ell_{\text {inf }} \cdot t\right\}$, takes on the $+\infty \delta_{0}$ initial trace.

Theorem 4.1. Let $f \in \mathcal{F}$, concave and such that $\mathrm{M}(f)=+\infty$. Then the special solution in (2.4) is an extended weak solution with infinite shock on $\Gamma=\left\{x=\ell_{\mathrm{inf}} \cdot t\right\}$, and takes on the initial data $\nu=+\infty \delta_{0}$ in the sense of Borel trace.

Proof. We already now that $\mathcal{U}$ is an extended entropy solution from the preliminary section. It remains to prove the initial trace. In fact, as $t \rightarrow 0, \mathcal{U}(x, t) \rightarrow 0$ locally uniformly in $\mathbb{R}_{*}$. Indeed, this comes from the fact that $\left(f^{\prime}\right)^{(-1)} \rightarrow 0$ as $\eta \rightarrow+\infty$ in this case. Thus the initial trace of $\mathcal{U}$ is zero outside $x=0$. Now, let $r>0$ fixed. If $t$ is sufficiently small, $B_{r}(0) \cap\left\{x=\ell_{\text {sup }} t\right\} \neq \emptyset$, but since $\mathcal{U}$ is not integrable near the shock curve $\Gamma$ (because $\mathrm{M}(f)=+\infty$ ),

$$
\int_{B_{r}(0)} \mathcal{U}(x, t) d x=+\infty \text { for any } t \text { small. }
$$

Thus in the sense of Borel trace, we have $\mathcal{U}(0)=+\infty \cdot \delta_{0}$.

Remark 4.2. We have proved also that the shock on $\Gamma$ is strong, i.e., $\mathcal{U}(t)$ is not integrable near $x=\ell_{\text {inf }} \cdot t$ for any $t>0$. This situation happens also in the parabolic version: a strong initial singularity remains as such for positive times, so that the equation is "lost" on the singularity curve.

Let us now investigate the fundamental solutions. The main result shows that fundamental solutions can be constructed as truncations of the self-similar solution:

Theorem 4.3. Let $f \in \mathcal{F}_{2}$. Then for any $c>0$, there exists a unique Entropic Fundamental Solution $u_{c}$ with mass $c$, given by:

$$
u_{c}(x, t)=\left\{\begin{aligned}
\mathcal{U}(x, t) & \text { if } x \geqslant \xi_{c}(t) \\
0 & \text { if } x<\xi_{c}(t)
\end{aligned}\right.
$$

where $\mathcal{U}$ is the Infinite Shock Solution and $\xi_{c}(t)>0$ is defined implicitly by the condition

$$
\forall t>0, \quad \int_{\xi_{c}(t)}^{\ell_{\text {sup }} t} U(x, t) d x=c .
$$


Proof. It is done as in the convex case, with some obvious modifications: first $\left(f^{\prime}\right)^{(-1)}$ is integrable near $\eta=\ell_{\text {sup }}$, while $\mathrm{M}(f)=\infty$ implies that for any $c>0$ and $t>0, \xi_{c}(t)$ is defined implicitly by (4.3). The positivity of $\xi_{c}$ is obvious, and the same calculation as in the convex case implies that

$$
\xi_{c}^{\prime}(t)=\frac{f(\mathcal{U})\left(\xi_{c}(t), t\right)}{\mathcal{U}\left(\xi_{c}(t), t\right)},
$$

which expresses here also the Rankine-Hugoniot condition on the curve, since this time, $u_{c}$ is zero at the left of the shock. The shock is entropic since the jump goes up as $x$ increases (remember that $f$ is concave in this section).

Remark 4.4. It should be noticed that the entropic fundamental solutions do not have any infinite shock, since the truncation by the shock curve $\xi_{c}$ avoids the singularities of $\mathcal{U}$ on the axis $\left\{x=\ell_{\text {inf }} \cdot t\right\}$ : by definition, $\xi_{c}(t)>\ell_{\text {inf }} \cdot t$.

Corollary 4.5. Let $f \in \mathcal{F}$, concave and such that $\mathrm{M}(f)=+\infty$. Then the limit of the entropic fundamental solutions as the mass $c$ goes to $\infty$ is exactly the special solution:

$$
\lim _{c \rightarrow \infty} u_{c}(x, t)=\mathcal{U}(x, t) .
$$

Moreover, the shock curves $\left\{x=\xi_{c}(t)\right\}$ converge to the infinite shock curve $\Gamma=\{x=$ $\left.\ell_{\text {inf }} \cdot t\right\}$.

The proof is exactly the same as in the convex case, with obvious modifications. Here also, all the shock curves $\operatorname{Graph}\left(\xi_{c}\right)$ remain between the lines $\left\{x=\ell_{\text {inf }} \cdot t\right\}$ and $\left\{x=\ell_{\text {sup }} \cdot t\right\}$.

\section{A Non-Existence Result}

We show below that if the total mass $\mathrm{M}(f)$ carried by $\left(f^{\prime}\right)^{(-1)}$ is bounded, then no fundamental solution can exist, whether the function $f$ is convex or concave. Before stating our results, let us mention that for instance

$$
f(x)=\int_{0}^{x} t^{-1 / 2} e^{-t} d t
$$

is concave, with $\ell_{\text {inf }}=0, \ell_{\text {sup }}=+\infty$, and satisfies

$$
\mathrm{M}(f)=\int_{0}^{\infty}\left(f^{\prime}\right)^{(-1)}(\eta) d \eta=-\int_{0}^{\infty} s f^{\prime \prime}(s) d s=\int_{0}^{\infty} e^{-s}\left(\frac{1}{2 s^{1 / 2}}+s^{1 / 2}\right)<\infty,
$$

so that the following discussion makes sense. We already pointed out that in the convex case, $\ell_{\text {sup }}=+\infty$ implies $\mathrm{M}(f)=+\infty$. Thus, for convex functions $f, \mathrm{M}(f)<$ $+\infty$ implies $\ell_{\text {sup }}<+\infty$. 
Theorem 5.1. Let $f \in \mathcal{F}$, either convex or concave with $\mathrm{M}(f)<+\infty$. Then the special solution $\mathcal{U}$ defined in (2.4) is not trivial. However, this solution carries the mass:

$$
\int_{\mathbb{R}^{N}} \mathcal{U}(x, t) d x=\mathrm{M}(f) \cdot t,
$$

hence $\mathcal{U}$ takes on the zero initial data in the sense of traces, although it has infinite shocks on one of the curves $\Gamma=\left\{x=\ell_{\mathrm{inf}} \cdot t\right\}$ or $\left\{x=\ell_{\mathrm{sup}} \cdot t\right\}$, according to the convexity of $f$.

Proof. We already know from the preliminary section that $\mathcal{U}$ is always defined under the assumptions $f \in \mathcal{F}$, convex or concave. Moreover, we have seen that an infinite shock occurs on one of the lines $\left\{x=\ell_{\text {inf }} \cdot t\right\}$ or $\left\{x=\ell_{\text {sup }} \cdot t\right\}$. These shock curves are always in accordance with the extended version of Rankine-Hugoniot condition that we introduced, so that $\mathcal{U}$ is always an extended entropy solution. However, since $\mathrm{M}(f)<\infty$, we can easily calculate

$$
\int_{\mathbb{R}} \mathcal{U}(x, t) d x=t \cdot \int_{\mathcal{I}}\left(f^{\prime}\right)^{(-1)}(\eta) d \eta=\mathrm{M}(f) \cdot t,
$$

Hence the result.

Remark 5.2. We should probably give some important explanations now:

i) In the convex case, the infinite shock curve $\Gamma$ cannot be located on the axis $\{t=0\}$ since $\mathrm{M}(f)<+\infty$ (indeed, $\ell_{\text {sup }}<+\infty$ as we recalled). Thus, in the set $\{0 \leqslant x \leqslant$ $\left.\ell_{\text {sup }} \cdot t\right\}, \mathcal{U}$ is zero, which explains why the initial trace of $\mathcal{U}$ is zero in the measure sense.

ii) In the concave case, the infinite shock curve can be located on the axis $\{x=0\}$, but since the total mass is finite, this means that the singularities are weak, i.e., $\mathcal{U} \rightarrow+\infty$ near $\Gamma$, but remains integrable in the $x$-variable near $\Gamma=\left\{x=\ell_{\text {sup }} \cdot t\right\}$.

iii) We see here a major distinction between the convex and concave cases, which avoids to simply make a change of variable $x \leftrightarrow t$. This is due to the fact that the initial trace makes a big difference between the $x$ and $t$ variables, since it consists in studying only spatial integrals of the solution.

Now, the total mass of $\mathcal{U}$ at time $t>0$ is exactly $\mathrm{M}(f) \cdot t$, which seems in contradiction with the conservation law. In fact, if $\mathcal{U}$ satisfies the equation in $Q_{+}$and $Q_{-}$, it does not in the whole set $\mathbb{R}^{2}$. Indeed, the presence of a forcing term located on the shock curve $\Gamma$ explains the non conservation of mass phenomenon. We shall prove this result in the simple case $f$ concave, $\ell_{\text {inf }}=0, \ell_{\text {sup }}=+\infty$ :

Proposition 5.3. Let $f \in \mathcal{F}$, concave with $\ell_{\mathrm{inf}}=0, \ell_{\mathrm{sup}}=+\infty$, and $\mathrm{M}(f)<+\infty$. Then $\mathcal{U}$ satisfies the following equation in the sense of distributions :

$$
\partial_{t} \mathcal{U}+\partial_{x} f(\mathcal{U})=\mathrm{M}(f) \cdot \delta_{0}(x) \otimes 1(t)
$$


Proof. Let $\varphi \in \mathcal{C}_{0}^{2}\left(\mathbb{R}^{2}\right)$ and $\varphi_{n} \in \mathcal{C}^{0}(\mathbb{R})$ be the sequence of functions defined as follows:

$$
\varphi_{n}(x)= \begin{cases}0 & \text { if }|x| \leqslant 1 / n \\ n(x-1 / n) & \text { if } 2 / n \leqslant x \leqslant 1 / n, \\ 1 & \text { if }|x| \geqslant 2 / n\end{cases}
$$

Since $\mathcal{U}$ is a continuous solution of the equation in $Q_{-}$and $Q_{+}$, we may use the test function $\varphi(x, s) \cdot \varphi_{n}(x)$ and make integrations by parts, which yields:

$$
-\int_{0}^{t} \int \mathcal{U} \varphi_{n} \partial_{t} \varphi-\int_{0}^{t} \int f(\mathcal{U}) \varphi_{n} \partial_{x} \varphi=n \cdot \int_{0}^{t} \int_{1 / n}^{2 / n} f(\mathcal{U}) \varphi .
$$

Let us consider the distribution $T_{n}:=n \chi_{n} f(\mathcal{U}(x / s))$, where $\chi_{n}$ is the characteristic function of the set $[1 / n ; 2 / n] \otimes 1(t)$. Then clearly the support of $T_{n}$ reduces to the axis $\{x=0\}$ as $n \rightarrow \infty$, so that in the limit there exists a measure $\lambda(t)$ such that,

$$
T_{n} \rightarrow \delta_{0}(x) \otimes \lambda(t)
$$

Finally, the measure $\lambda$ can be calculated as follows: if we take $\varphi \equiv 1$, after integrating by parts in time we obtain

$$
\int \mathcal{U}(t) \varphi_{n}=\int_{0}^{t} \int T_{n}(x, s),
$$

and we know that the total mass carried by $\mathcal{U}$ at time $t$ is $\mathrm{M}(f) \cdot t$. Thus,

$$
\int_{0}^{t} \int T_{n}(x, s) \rightarrow \lambda(0, t)=\mathrm{M}(f) \cdot t,
$$

which implies that $\lambda(t)=\mathrm{M}(f) 1(t)$. Finally, coming back to (5.3), we obtain in the limit:

$$
-\int_{0}^{t} \int \mathcal{U} \partial_{t} \varphi-\int_{0}^{t} \int f(\mathcal{U}) \partial_{x} \varphi=\mathrm{M}(f) \int_{0}^{t} f(\mathcal{U}(0, s)) \varphi(0, s) d x
$$

which means exactly (5.1).

As was said, in the general cases, one can show by the same method that the forcing term is located on the shock curve $\Gamma$. Hence, $\mathcal{U}$ is always a solution of the equation

$$
\partial_{t} \mathcal{U}+\partial_{x} f(\mathcal{U})=\mathrm{M}(f) \cdot \mu_{\Gamma}
$$

where $\mu_{\Gamma}$ stands for the measure $\delta_{0}\left(x-\ell_{\text {inf }} \cdot t\right)$ or $\delta_{0}\left(x-\ell_{\text {sup }} \cdot t\right)$, according to the convexity of $f$. The proof follows the same lines as the simple case above.

Now, we prove below that fundamental solutions do not exist in the present situation. We shall also prove the result in the concave case, when $\ell_{\text {inf }}=0, \ell_{\text {sup }}=+\infty$ 
for the sake of simplicity, the other cases requiring only minor modifications. Before, we need the following approximation Lemma:

Lemma 5.4. Let $f$ be concave with $\mathrm{M}(f)<+\infty$ and $\ell_{\mathrm{inf}}=0, \ell_{\mathrm{sup}}=+\infty$. Then there exists a sequence $f_{\varepsilon}$ satisfying the following conditions: $f_{\varepsilon} \in \mathcal{F}, \mathrm{M}\left(f_{\varepsilon}\right)=+\infty, f_{\varepsilon}$ is concave, nondecreasing, $\left(f_{\varepsilon}^{\prime}\right)^{(-1)}$ is integrable near $+\infty$, and decreases to $\left(f^{\prime}\right)^{(-1)}$ as $\varepsilon$ decreases to 0 .

Proof. For $f$ as above, we may consider the function

$$
g_{\varepsilon}: u \mapsto\left(f^{\prime}\right)^{(-1)}(u)+\varepsilon g(u),
$$

where $g \in \mathrm{C}^{1}(0, \infty)$ is nonincreasing, $g \geqslant 0$, integrable near $u=+\infty$ and $g(u) \sim 1 / u$ near $u=0$. Then clearly $g_{\varepsilon}$ is nonincreasing on $(0, \infty)$ with $g(0)=+\infty, g(\infty)=0$ so that

$$
f_{\varepsilon}: u \mapsto \int_{0}^{u}\left(g_{\varepsilon}\right)^{(-1)}(s) d s
$$

is well-defined and satisfies the conditions of the statement: $f_{\varepsilon}$ is concave, $\mathrm{M}\left(f_{\varepsilon}\right)=$ $+\infty$ since $g$ is not integrable near 0 , and $g_{\varepsilon}=\left(f_{\varepsilon}^{\prime}\right)^{(-1)}$ decreases to $\left(f^{\prime}\right)^{(-1)}$ as $\varepsilon \rightarrow 0$

Now, for any $\varepsilon>0$, the fundamental solution $u_{c}^{\varepsilon}$ with mass $c>0$ exists. But we prove that as $\varepsilon \rightarrow 0$, these fundamental solutions converge to a solution in $\left(\mathbb{R}^{+}\right)^{2}$ which is not a fundamental solution.

Proposition 5.5. Let $f$ be concave with $\mathrm{M}(f)<+\infty$ and $\ell_{\mathrm{inf}}=0, \ell_{\mathrm{sup}}=+\infty$. For any $\varepsilon>0$, there exists a unique fundamental solution $u_{c}^{\varepsilon}$ with mass $c>0$ associated with the flux $f_{\varepsilon}$. Then as $\varepsilon \rightarrow 0$, the sequence $u_{c}^{\varepsilon}$ converges to the following function:

$$
\lim _{\varepsilon \rightarrow 0} u_{c}^{\varepsilon}= \begin{cases}\left(f^{\prime}\right)(-1)(x / t) & \text { if } x \geqslant \xi_{c}(t) \\ 0 & \text { if } x<\xi_{c}(t),\end{cases}
$$

where $\xi_{c}(t)$ is defined as follows: $\xi_{c}(t)=0$ for $t \in[0, c / \mathrm{M}(f)]$, and

$$
\forall t>c / \mathrm{M}(f), \quad \int_{\xi_{c}(t)}^{\infty}\left(f^{\prime}\right)^{(-1)}(x / t) d x=c .
$$

Since this limit takes the zero initial trace in the sense of measures, the fundamental solutions do not exist (in this limit sense) for the function $f$.

Proof. For any $\varepsilon>0$, since $\mathrm{M}\left(f_{\varepsilon}\right)=+\infty$, we know exactly the form of the fundamental solution with mass $c>0$ :

$$
u_{c}^{\varepsilon}=\left\{\begin{array}{rll}
g_{\varepsilon}(x / t) & \text { if } & x \geqslant \xi_{c}^{\varepsilon}(t) \\
0 & \text { if } & x<\xi_{c}^{\varepsilon}(t)
\end{array}\right.
$$

where $\xi_{c}^{\varepsilon}(t)>0$ is defined implicitly by the condition

$$
\forall t>0, \quad \int_{\xi_{c}^{\varepsilon}(t)}^{+\infty} g_{\varepsilon}(x / t) d x=c .
$$


Since $g_{\varepsilon}$ decreases, the shock curve $\xi_{c}^{\varepsilon}$ has to go closer to the $t$-axis, that is, the sequence $\varepsilon \rightarrow \xi_{c}^{\varepsilon}(t)$ is nonincreasing for fixed $t>0$.

i) Let us prove that for any $t<c / \mathrm{M}(f), \xi_{c}^{\varepsilon}(t) \rightarrow 0$. Remember that the total mass carried by $\left(f^{\prime}\right)^{(-1)}$ at time $t>0$ is exactly $\mathrm{M}(f) \cdot t$, and let us assume that for $t<c / \mathrm{M}(f), \xi_{c}^{\varepsilon}(t) \rightarrow \xi_{c}(t)>0$. Then from

$$
c=\int_{\xi_{c}^{\varepsilon}(t)}^{+\infty}\left\{\left(f^{\prime}\right)^{(-1)}(x / t)+\varepsilon \cdot g(x / t)\right\} d x,
$$

it follows as $\varepsilon \rightarrow 0$ that (remember that $g$ is integrable near $+\infty$ )

$$
c=\int_{\xi_{c}(t)}^{+\infty}\left(f^{\prime}\right)^{(-1)}(x / t)=\mathrm{M}(f) \cdot t,
$$

which is impossible since $t<c / \mathrm{M}(f)$. Thus, it is necessary that $\xi_{c}^{\varepsilon}(t)$ converge to 0 for such $t$, so that for $0<t<c / \mathrm{M}(f)$, the limit function is the self-similar solution $\left(f^{\prime}\right)^{(-1)}(x / t)$ in $\left(\mathbb{R}_{+}\right)^{2}$.

ii) Now for $t \geqslant c / \mathrm{M}(f)$, the total mass carried by $\left(f^{\prime}\right)^{(-1)}$ is $\mathrm{M}(f) \cdot t$, which is greater than $c$. Thus the shock curve $\xi_{c}^{\varepsilon}(t)$ decreases to a shock curve $\xi_{c}(t)$ defined implicitly by (5.11).

iii) Of course, the initial trace of the limit solution is zero since the total mass carried by $\left(f^{\prime}\right)^{(-1)}$ is $\mathrm{M}(f) \cdot t \rightarrow 0$ as $t \rightarrow 0$. Thus, the limit function is not a fundamental solution, which ends the proof.

Remark 5.6. It can be shown that the limit solution $\lim _{\varepsilon \rightarrow 0} u_{c}^{\varepsilon}$ converges to the selfsimilar solution in the whole set $\left(\mathbb{R}_{+}\right)^{2}$ as $c \rightarrow+\infty$, by studying the shock curve. Indeed, $\xi_{c}(t)=0$ for any $0<t<c / \mathrm{M}(f)$, so that in the limit,

$$
\lim _{c \rightarrow \infty} \xi_{c}(t)=0 \quad \forall t>0 .
$$

\section{Infinite Shocks obtained by Viscous Approximations}

We go back to concave fluxes with mass $\mathrm{M}(f)=+\infty$, in which situation infinite shocks may occur as we saw in Section 4. Of course, such solutions cannot be constructed by the usual vanishing viscosity method, since the singularity is strong. Indeed, strong singularities (i.e., not integrable singularities) are not allowed for the Heat Equation.

However, strong singularities are allowed in the fast-diffusion case $[2,3]$, so that one might wonder whether an Infinite Shock Solution can be obtained as the limit as $\varepsilon \rightarrow 0$ of the following approximate problems :

$$
\left\{\begin{array}{c}
u_{t}+f(u)_{x}=\varepsilon f(u)_{x x} \\
u(0)=+\infty \delta_{0}
\end{array}\right.
$$

We shall now prove the result in the power-case. The case of non power fluxes requires more work since the separate-variable form is not available. In the power-case 
$f(u)=u^{m}, 0<m<1$, the Infinite Shock Solution has the separate-variable form $\mathcal{U}(x, t)=t^{1 /(1-m)} \psi(x)$, where $\psi$ satisfies:

$$
\left\{\begin{array}{cc}
\psi^{\prime}+c(m) \psi^{q}=0 & \text { if } x>0 \\
\psi=0 & \text { if } x<0
\end{array}\right.
$$

with $q=1 / m>1, c(m)=1 /(1-m)$. We can solve explicitly this equation, which yields

$$
\psi(x)=c_{1}|x|^{-1 /(q-1)}, \quad c_{1}=q^{-1 /(q-1)} .
$$

Thus, in the power case, we prove:

Proposition 6.1. Let $f(u)=u^{m}, 0<m<1$. Then the Infinite Shock Solution $\mathcal{U}$ is the limit as $\varepsilon \rightarrow 0$ of solutions of (6.1).

Proof. We consider the approximate elliptic equation

$$
-\varepsilon \psi_{\varepsilon}^{\prime \prime}+\psi_{\varepsilon}^{\prime}+c(m) \psi_{\varepsilon}^{q}=0 \quad x \in \mathbb{R},
$$

and notice that $t^{1 /(1-m)} \psi_{\varepsilon}(x)$ is a solution of (6.1) provided we can solve this last equation with infinite data for $x=0$. In this case, we obtain a solution with standing strong singularity, a kind of solutions that were studied in detail by the author and J.L. Vázquez in $[2,3]$.

We will consider separately the cases $x>0$ and $x>0$ :

- For $x<0$, any solution of (6.2) is bounded by the solution of $-\varepsilon \psi^{\prime \prime}+c(m) \psi^{q}=0$ which explodes at $x=0$. The solution $\psi_{*}$ of this last equation is explicit,

$$
\psi_{*}(x)=c_{2}(\varepsilon)|x|^{-2 /(q-1)}, \quad c_{2}(\varepsilon)=\left(\frac{2(q+1) \varepsilon}{q(q-1)}\right)^{1 /(q-1)} .
$$

Thanks to this bound, we can construct a solution of the approximate problem for $x<0$ by standard methods, choosing the value $\psi_{\varepsilon}(0)=c$ and letting $c$ increase to $+\infty$ : we obtain a solution $\psi_{\varepsilon}$ of (6.2) for $x>0$ with value $+\infty$ at $x=0$. The bound above also shows that for $x>0, \psi_{\varepsilon} \rightarrow 0$ as $\varepsilon \rightarrow 0$.

- For $x>0$ we use the same method: we first solve the problem with data $\psi_{\varepsilon}(0)=c$, $c>0$ and let $c$ increase to $+\infty$. In order to get a solution in the limit, we need a bound above. To this end, we construct a super-solution of the form

$$
w(x)=w_{\alpha, \beta}(x)=\alpha|x|^{-\frac{2}{q-1}}+\beta|x|^{-\frac{1}{q-1}},
$$

with a suitable choice of the parameters $\alpha, \beta>0$. Using the convexity inequality $(a+b)^{q} \geqslant a^{q}+b^{q}$ for $a, b>0, q>1$, straightforward calculations give:

$$
-\varepsilon w^{\prime \prime}+w^{\prime}+c(m) w^{q} \geqslant c_{\alpha}|x|^{-\frac{2 q}{q-1}}-c_{\alpha, \beta}(x)+c_{\beta}|x|^{-\frac{q}{q-1}} .
$$


with

$$
\begin{gathered}
c_{\alpha}=\left[c(m) \alpha^{q}-\frac{2 \alpha \varepsilon(q+1)}{(q-1)^{2}}\right], \quad c_{\beta}=\left[c(m) \beta^{q}-\frac{\beta}{q-1}\right], \\
c_{\alpha, \beta}(x)=\frac{2 \alpha}{q-1}|x|^{-\frac{q+1}{q-1}}+\frac{\beta \varepsilon q}{(q-1)^{2}}|x|^{-\frac{2 q-1}{q-1}} .
\end{gathered}
$$

First, we may choose $\alpha$ and $\beta$ big enough (independently of $\varepsilon<1$ ) so that $c_{\alpha}, c_{\beta}>0$. Moreover, since

$$
|x|^{-\frac{q+1}{q-1}},|x|^{-\frac{2 q-1}{q-1}} \leqslant|x|^{-\frac{2 q}{q-1}}+|x|^{-\frac{q}{q-1}},
$$

we can also choose $\alpha, \beta$ independently of $\varepsilon<1$ so that

$$
c_{\alpha, \beta}(x) \leqslant c_{\alpha}|x|^{-\frac{2 q}{q-1}}+c_{\beta}|x|^{-\frac{q}{q-1}},
$$

hence $w$ is a super-solution of the problem, with value $+\infty$ at $x=0$. This gives a universal bound so that the limit as $c \rightarrow \infty$ exists and it is the solution $\psi_{\varepsilon}$ we were looking for. Moreover, $\psi_{\varepsilon} \leqslant w$.

Let us now study the limit as $\varepsilon \rightarrow 0$, and let us first notice that $\psi$ itself is a sub-solution of $(6.2)$, so that $\psi(x) \leqslant \psi_{\varepsilon}(x) \leqslant w_{\alpha, \beta}(x)$ for $x>0$. Since $w_{\alpha, \beta}$ may be chosen independently of $\varepsilon \rightarrow 0$, the limit $\psi_{0}=\lim _{\varepsilon \rightarrow 0} \psi_{\varepsilon}$ is well-defined (up to extraction) and satisfies the same comparison. Moreover, as $\varepsilon \rightarrow 0, \alpha$ and $\beta$ may be chosen close to their limit values $\alpha_{*}, \beta_{*}$ defined by

$$
c(m) \alpha^{q}=\frac{2 \alpha}{q-1}, \quad c(m) \beta^{q}=\frac{\beta}{q-1},
$$

which ensure that $c_{\alpha}, c_{\beta}$ remain positive and that $c_{\alpha, \beta}$ is still dominated by the positive terms. Notice that precisely, $\beta_{*}=c_{1}$, so that we obtain in the limit:

$$
\psi(x)=c_{1}|x|^{-\frac{1}{q-1}} \leqslant \psi_{0}(x) \leqslant w_{\alpha_{*}, \beta_{*}}(x)=\alpha_{*}|x|^{-\frac{2}{q-1}}+c_{1}|x|^{-\frac{1}{q-1}}, \quad x>0 .
$$

Now, if $k>0$, then $v_{k}(x)=k^{-1 /(q-1)} \psi_{\varepsilon}(x / k)$ satisfies the equation

$$
-\frac{\varepsilon}{k} v_{k}^{\prime \prime}+v_{k}^{\prime}+c(m) v_{k}^{q}=0, \quad x>0 .
$$

Hence, the $\psi_{\varepsilon}$ are deduced from one another by rescaling, so that standard arguments show that the limit as $\varepsilon \rightarrow 0$ takes the self-similar form:

$$
\psi_{0}(x)=c|x|^{-1 /(q-1)},
$$

for some $c>0$. Now using (6.3), we easily see that necessarily $c=c_{1}$, that is, $\psi_{0} \equiv \psi$. Thus the solution of (6.1) converges to the Infinite Shock Solution. 


\section{Conclusions and Comments}

In this paper, we have generalized the power-case $f(u)=u^{m}$, where we have explicit formulas for fundamental solutions to non power functions $f$. We have extended the notion of entropy solution in order to deal with solutions having infinite shocks. In this setting, we have proved that even in the non-Lipschitz case, fundamental solutions do exist provided some requirements on $f$ are fulfilled, and given their limit as the mass goes to $+\infty$. In some other cases, those solutions do not exist. We now list below a number of questions that have not been addressed in this paper:

Generalization. We have restricted our study to functions $f$ convex or concave in order to have $\left(f^{\prime}\right)^{(-1)}$ well-defined on some open interval $\mathcal{I}$. However, it is possible to handle the case of multi-valued $\left(f^{\prime}\right)^{(-1)}$ by selecting a determination in each subinterval of $\mathcal{I}$. This leads to several special solutions $\mathcal{U}$ according to the choice of $\left(f^{\prime}\right)^{(-1)}$. However, discontinuities along the curves $\{x=\ell \cdot t\}$ have to fulfill the Rankine-Hugoniot condition, so that not all the choices are possible. In this setting, we can construct fundamental solutions provided there exists a choice of $\left(f^{\prime}\right)^{(-1)}$ with infinite mass.

Existence for measures. In the case when fundamental solutions exist, one may ask if any measure would be admissible as an initial data. The lack of compactness does not allow to use the proof of Liu and Pierre [11] in general, but probably if Dirac masses are allowed, any other measure is also since Deltas are the most singular measures.

Non existence for measures. In the case when no fundamental solution can exist, is it possible however to construct solutions for a class of diffuse measures ? Maybe a capacity-type criterion could be involved.

Long-time Behaviour. Probably the fundamental solutions describe also the longtime behaviour in the non-Lipschitz case, at least for finite shocks. But if infinite shocks occur, then the asymptotic behaviour could be given by the Infinite Shock Solution, as it is the case for the fast-diffusion equation (see [3]). Another question concerns the asymptotic behaviour when no fundamental solution exist.

\section{References}

[1] E. Chasseigne, Initial trace for a porous medium equation: I. The strong absorption case, Annali di Matematica Pura ed Applicata 179 (2001), 413-458.

[2] E. Chasseigne, Classification of Razor Blades to the filtration equation, Journal of Differential Equations 187 (2003), 72-105.

[3] E. Chasseigne, J.L. Vázquez, Theory of extended solutions for fast-diffusion equations. Radiation from singularities, Archive for Rational Mechanics and Analysis 164 (2002), 133-187. 
[4] E. Chasseigne, J.L. Vázquez, Sets of admissible initial data for porous medium equations with absorption, Electron. J. Differ. Equ. Conf., 8 (2002), 53-83.

[5] C.M. Dafermos, Hyperbolic conservation laws in continuum physics, Grundlehren der Mathematischen Wissenschaften 325, Springer (2000).

[6] B.E.J. Dahlberg, C. Kenig, Nonnegative solutions the of the initial-Dirichlet problem for generalized porous medium equation in cylinders, Jl. Amer. Math. Soc. 1 (1988), 401-412.

[7] E. Godlewski, P.A. Raviart, Hyperbolic systems of conservation laws, Ellipses, Paris (1991).

[8] F. Guarguaglini, Singular solutions and asymptotic behaviour for a class of conservation laws with absorption, Commun. Partial Differ. Equations, 20 (1995), 1395-1425

[9] Y. Hongjun, Source-type solutions of a singular conservation law with absorption. Nonlinear Anal., Theory Methods Appl. 32, 467-492 (1998).

[10] S.N. Kruzhkov, First-order quasilinear equations in several independent variables, Math. USSR-Sb. 64 (1977), 47-57.

[11] T.P. Liu, M. Pierre, Source solutions and asymptotic behaviour in conservation laws, Jl. Diff. Equ 51 (1984), 419-441.

[12] M. Marcus, L. Véron, The boundary trace of positive solutions of semilinear elliptic equations: the subcritical case, Arch. Rat. Mech. An. 144 (1998), 201-231.

[13] M. Marcus, L. Véron, Initial trace of positive solutions of some nonlinear parabolic equations, Commun. Partial Differ. Equations 24 (1999), 1445-1499.

[14] J.L. Vázquez, L. Véron, Different kinds of singular solutions of nonlinear parabolic equations, Nonlinear Problems in Applied Mathematics (volume in honor of Ivar Stakgold on his 70th birthday), T.S. Angell et al. eds., SIAM, Philadelphia, 1996, pp. 240-249.

[15] L. Véron, Singularities of solutions of second order quasilinear equations, Pitman Research Notes in Mathematics 353 (1996). 